\title{
Cancer Manipulation of Host Physiology- Lessons from Pancreatic
}

\section{Cancer}

Constantinos P. Zambirinis, MD, MRes ${ }^{1,2}$, George Miller, MD ${ }^{3,4}$

${ }^{1}$ Hepatopancreatobiliary Service, Department of Surgery, Memorial Sloan Kettering Cancer Center, New York, NY 10065

${ }^{2}$ Department of Surgery, Harlem Hospital, Columbia University Medical Center, New York, NY 10037

Departments of ${ }^{3}$ Surgery and ${ }^{4}$ Cell Biology, New York University School of Medicine, New York, NY 10016

Keywords: Pancreatic ductal adenocarcinoma; inflammation; cancer-associated fibroblasts; stroma; immunity

Correspondence: george.miller@nyumc.org (G. Miller) 


\begin{abstract}
Homeostasis is a fundamental property of living organisms enabling the human body to withstand internal and external insults. In several chronic diseases, and especially in cancer, many homeostatic mechanisms are deranged. Pancreatic cancer, in particular, is notorious for its ability to invoke an intense fibro-inflammatory stromal reaction facilitating its progression and resistance to treatment. In the past decade, a number of seminal discoveries have elucidated previously unrecognized modes of commandeering the host's defense systems. Here, we review novel discoveries in pancreatic cancer immunobiology and attempt to integrate the notion of deranged homeostasis in the pathogenesis of this disease. We also highlight areas of controversy and obstacles that need to be overcome, hoping to further our mechanistic insight into this malignancy.
\end{abstract}




\section{Primer: Cancer Thrives Amongst Chaos}

The human body responds to aberrations of normal physiology in numerous ways, attempting to maintain or re-establish balance - this is the essence of homeostasis. The immune system and associated cascades such as the complement system and various tissue regeneration processes, is a vital component of homeostatic responses to tissue stress. If dysregulated, it may perpetuate disease rather than abate it. This holds true for almost all pathologies, including cancer (Key Figure, Figure 1). The importance of understanding homeostatic responses in carcinogenesis stems from two critical determinants in the course of neoplastic diseases: first, that homeostatic responses may be subverted in ways that propagate - rather than counteract carcinogenesis; and second, that anti-cancer therapies may be rendered ineffective by the bystander effects of homeostatic responses. The timeliness of this topic derives from the recent explosion in high-throughput technologies that can generate an immense amount of information pertaining to the biology of a given tumor. These have brought tremendous excitement and have heralded the era of personalized medicine through the identification of pathways specifically deranged in cancer cells; yet, they often neglect, or fail to take into consideration the nontransformed aspect of a tumor, or "stroma", which results from aberrant homeostatic responses. Indeed, it is vital, not only to the pathophysiology of cancer, but also to the mechanisms of tumor resistance to many novel targeted therapies.

Pancreatic ductal adenocarcinoma (PDAC; the most common form of pancreatic cancer) is probably the best example of an inflammation-driven neoplastic process [1]. Notably, its incidence is climbing even though our ability to treat it effectively has improved only meagerly in the past few decades. As such, it is projected that PDAC will soon become the second most lethal cancer in the developed world [2]. PDAC is remarkable for its overwhelming stromal 
infiltrate and the resultant fibrotic reaction (also known as "desmoplasia") - which exemplifies how homeostatic responses can be detrimental when curtailed by cancer. In the past decade, a number of seminal studies have elucidated previously under-appreciated aspects of the pathogenesis of PDAC-associated stromal reaction and its fundamental role in pancreatic carcinogenesis. However, they have also fueled considerable debate since some of the most recent evidence contradicts earlier doctrines (such as the anti-tumorigenic role of Toll-like receptors). In this review, we attempt to bring an alternative perspective to pancreatic carcinogenesis (and by extension, to all solid tumors), by focusing on the non-transformed aspect of the tumor that is fueled by aberrant homeostatic responses to tissue stress, characterized by dysregulated inflammatory responses and failed regeneration, thus affecting the body as a whole.

\section{Tissue Stress, Inflammation and Carcinogenesis.}

Inflammation involves the coordinated activation of several components including the immune system, the complement system, the coagulation cascade, and the wound healing/tissue regeneration program which crosstalk to each other in a coordinated manner. The ultimate goal is to eliminate the noxious stimuli that lead to the initiation of an inflammatory response and restore balance, ideally with reversal of the compromised function of affected tissues. Therefore, activation of tissue resident immune cells (e.g. macrophages, intraepithelial lymphocytes) and other supportive cells, as well as the sequential recruitment of innate (e.g. neutrophils, mast cells, monocytes, NK cells, $\boldsymbol{\gamma} \boldsymbol{\delta} \mathbf{T}$ cells) and adaptive immune cells (B and T lymphocytes) are required to fight off exogenous (microbes) or endogenous (e.g. stressed/transformed cells) offenders. At the same time, immune and other supportive cells release soluble mediators, including cytokines such as interleukin-6 (IL-6) and tumor necrosis factor (TNF), that activate complementary loco- 
regional responses as well as systemic programs to conserve energy and create an organism-wide state that is less conducive to infection. For example, phenomena such as febrile reactions, decreased iron availability, and altered hepatic metabolism, among others, aim to protect the host from side effects of tissue stress, and prevent or curtail various deleterious effects. The complement system is activated early during the inflammatory response and contributes to pathogen elimination directly via lysis of bacteria, and indirectly, via opsonization, while some molecular intermediates generated during these processes recruit immune and other cells to sites of inflammation [3]. The adjacent endothelial cells and the coagulation cascade are also activated and, apart from a hemostatic response, they serve to recruit additional immune cells as well as platelets and fibroblasts to promote the healing response.

Viewed from a systems dynamics perspective, inflammation may be simplified in a homeostatic circuit (Fig. 2) [4]. Disruption of normal cellular function by an 'inducer' (e.g. traumatic injury) is perceived by a 'sensor' (e.g. innate immune cells) as a deviation from the norm, or set point. For example, cellular stress causes release of damage-associated molecular patterns (DAMPs) that bind pattern-recognition receptors (PRRs) that activate innate immune cells $[5,6]$. The sensor in turn will generate a 'signal' (e.g. pro-inflammatory cytokines) that will activate an 'effector' cell (e.g. lymphocytes), which in turn, will attempt to alleviate the inducer in order to restore the deranged variable within the normal range - for instance, by clearing off necrotic debris and repairing the injured tissue.

A very important aspect of all these processes is an ability to self- and cross-regulate the degree of activation for each component in order to prevent unnecessary propagation of inflammation and thus, result in collateral damage $[4,7,8]$. Almost every arm of the homeostatic circuit can set off negative feedback loops that either directly inhibit the effectors, or modulate 
the threshold of activation ('gain') of both sensors and effectors. The end result is the organized resolution of the inflammatory response and the minimization of harmful off-target effects . When these pathways malfunction and do not obey the laws of negative feedback and homeostasis, chronic inflammation ensues, and can be more detrimental than beneficial, leading to pathological consequences such as fibrosis, loss of organ function, and carcinogenesis (Fig. 1). The unrestrained propagation of cancer-associated fibro-inflammatory elements that originate in a host's physiologic responses to injury, have led to the decades-old quip that tumors are "wounds that do not heal" $[9,10]$.

In the majority of cases, pancreatic cancer is characterized by a prominent inflammatory response $[1,11]$. However, this type of inflammation presents considerable differences from the acute inflammation that accompanies wound healing: it is self-sustaining, unregulated, and permissive to cancer cell development $[12,13]$. Thus, even though pancreatitis is a wellrecognized risk factor for pancreatic cancer development, most patients with PDAC have no clinical history of overt pancreatitis. [14].

Numerous studies have established the importance of chronic inflammation in pancreatic carcinogenesis and progression [12]. The interplay between inflammation and cancer-initiating mutations is particularly interesting. Kras mutations are present in the majority of PDAC cases as well as in a significant percentage of pancreatic intraepithelial neoplasia (PanIN) lesions, even at early stages (over 30\% of PanIN 1 harbor mutant Kras) [15]. Work done in wellvalidated genetically-engineered mouse models has revealed that mutant Kras not only initiates the stepwise progression from normal epithelium to acinar-to-ductal metaplasia (ADM), PanIN and invasive cancer, but also promotes immune cell infiltration through various mechanisms (reviewed in $[13,16]$ ), including the release of pro-inflammatory cytokines IL-1 $\alpha$ 
and IL-6 and chemoattractants such as granulocyte-monocyte colony stimulating factor (GMCSF). In both rodents and humans, pancreatic inflammation also cooperates with Kras mutations to hyperactivate downstream pathways such as nuclear factor kappa B (NF-kB), signal transducer and activator of transcription 3 (STAT3), and cyclooxygenase-2 (COX2), which in turn, can accelerate carcinogenesis through an autocrine/paracrine feedback loops, release additional pro-inflammatory mediators, and further perpetuate inflammation (Figures $\mathbf{3}$ and $\mathbf{4}$ ) $[13,16]$. We highlight several examples of homeostatic perturbations that sustain the protumorigenic inflammatory response that accompanies PDAC.

\section{Imbalance of Soluble Mediators}

Stressed cells, whether transformed or not, release DAMPs that bind PRRs and initiate or perpetuate inflammation. We and others have shown that the PDAC tumor microenvironment (TME) is rich in DAMPs, which bind Toll-like receptors (TLRs) and other PRRs such as the receptor for advanced glycation end-products (RAGE) in both mice and humans [13, 17-20]. In PDAC mouse models, ligation of several TLRs dramatically accelerates pancreatic oncogenesis through diverse mechanisms involving immune cell modulation as well as activation of pancreatic stellate cells (PSC) $[17,18,21]$. Other PRRs such as C-type lectin receptors (CLRs) also appear to have important roles. For instance, in both murine and human PDA, necroptotic epithelial cells release the histone deacetylase complex subunit SAP130 that acts as a DAMP by ligating Mincle -- a CLR found on various myeloid cells -- to promote suppression of antitumor immunity [19]. Disruption of the necroptotic pathway by genetic deletion or pharmacological inhibition of key signaling intermediates was shown to protect mice from pancreatic carcinogenesis, as did genetic ablation of Mincle[19]. 
Besides, PRRs can bind microbial byproducts that may originate from local or distant sites. Recent work has highlighted the role of oral microbial dysbiosis (i.e. imbalance of commensal flora and potentially pathogenic microorgansisms) in pancreatic carcinogenesis [13, $22,23]$. In a prospective human study featuring bacterial DNA profiling, overrepresentation of Neisseria elongata and Streptococcus mitis in saliva were strongly associated with PDAC [22]. Further, bacteria can directly access the pancreas. We found that orally administered Streptococcus mutans can reach the pancreas in mice, while a recent study identified Fusobacterium species in pancreatic tumor tissue and their presence correlated with higher mortality rates $[18,24]$. Our ongoing investigations suggest that the intestinal microbiome is also deranged in PDAC patients and contributes to perturbations of the immune infiltrate, while germ-free mice are protected from pancreatic carcinogenesis secondary to immunogenic reprogramming of the TME (unpublished work).

Transformed pancreatic epithelial cells release a wide array of soluble factors, including cytokines (such as IL-1 $\beta$, IL-6, IL-11, and TNF [18, 25, 26]), various chemokines (e.g. CCL2, CCL5, CXCL1, CXCL2, CXCL12 [19, 27-30]), and growth factors (such as GM-CSF and TGF$\beta[31,32])$, with profound effects on immune cell infiltration, activation status, as well as skewing towards cellular phenotypes that support tumor growth and immune escape (reviewed in the next section; Fig. 4). Some of the above are also released by non-transformed stressed cells [12]. The tumor-derived soluble factors often act in concert with each other, or with mediators derived from stromal cells, resulting in non-physiologic effects such as the generation of feedforward loops that sustain the inflammatory reaction and prevent homeostasis [26, 33, 34]. For example, pancreatic epithelial cells with Kras mutations recruit myeloid cells that secrete high amounts of IL-6; the latter activates STAT3 on epithelial cells and upregulates pro-proliferative 
and anti-apoptotic molecules as well as extracellular matrix (ECM) modulating enzymes such as matrix metallopeptidase 7 (MMP7). This ultimately promotes PDAC progression and aggressiveness [26, 33].

The CXCL12-CXCR4 is one of the most well studied chemokine axes in multiple types of cancer $[35,36]$. Both ligand and receptor are significantly upregulated on cancer cells as well as various other stromal cell types, and have important roles in bidirectional tumor-stroma communication in human PDAC, including promotion of tumor growth and invasion, enhancement of the cancer-associated fibroblast (CAF) compartment, and maintenance of intratumoral immunosuppression [36-38]. Intriguingly, it was recently implicated in neural invasion of PDAC: specifically, human peri-pancreatic Schwann cells were found to upregulate CXCR4 and CXCR7 in response to pancreatic cancer and associated hypoxia [30]. Cancer cells secrete CXCL12 which attracts peri-pancreatic nerves to infiltrate early PDAC lesions, rendering them less-responsive to pain [30]. Although this may be a defense mechanism to shield the patient from the intractable pain of pancreatic cancer-related neural invasion, it is clearly hijacked by cancer cells to promote loco-regional tumor dissemination [30].

Our understanding of the role of specific soluble mediators, particularly chemokines, is hampered by three important factors: First, several chemokines exhibit a great degree of redundancy, such that even if one is blocked, others can compensate for its absence. Second, chemokines exhibit promiscuity, acting on multiple receptors agonistically or antagonistically. Once again, targeting a single receptor might be insufficient to abrogate their effects. Lastly, even though many of the chemokines are conserved across mammalian species, some exhibit considerable structural and/or functional differences between humans and rodents, or may even be completely absent. One such example is CXCL8/IL-8, which is only present in humans and 
has important functions in recruitment of innate immune cells and angiogenesis, among others [39]. In conclusion, PDAC is characterized by profound imbalances of soluble mediators that have diverse and often overlapping functions. Therefore, investigations employing blockade of such promiscuous pathways should always take the above into consideration. Moreover, combination therapies against these mediators may be a better strategy for developing novel PDAC therapeutics.

\section{Immune Cell Perturbations}

The failure of the homeostasis of the immune system in the context of cancer development has been summarized in the concept of cancer immunoediting: Usually, the immune system successfully eradicates transformed cells (elimination). However, every once in a while cancer cells find ways to evade killing and persist in a "stealth mode" (equilibrium). As they accumulate additional mutations, the transformed cells that remain undetectable by cytotoxic immune effectors and can thrive in the pro-inflammatory tumor milieu are enriched and eventually prevail (escape). In pancreatic oncogenesis, the dynamics of immune cells are much more complex, with different cell types having multifaceted roles.

Myeloid cells such as neutrophils and macrophages have key roles in inflammatory diseases, including pancreatitis. Neutrophils - the predominant cell type involved in the early phases of acute inflammation - have been shown to contribute to pancreatic carcinogenesis and immunosuppression [11]. Chemokines such as CXCL5 and CXCL2 released by cancer cells and stromal cells, respectively, bind CXCR2 and attract neutrophils to the TME in human and murine PDAC [29]. Using murine PDAC cells, CXCL5 was shown to be upregulated in response to Kras mutations in a NF-kB-dependent manner in vitro. Moreover, genetic ablation of CXCR2 in 
a genetically-engineered mouse model of PDAC led to decreased neutrophil infiltration in tumors and augmented antitumor cytotoxic T cell immune responses, thus establishing a protumorigenic role for tumor-associated neutrophils (TANs) in PDAC. [29].

Macrophages arrive to sites of inflammation after neutrophils and persist longer [40]; indeed, they are one of the main cell types involved in chronic inflammation $[11,41]$. They infiltrate tumors in response to DAMPs and other danger signals are released secondary to tissue stress and hypoxia (which might be a consequence of chemo-/radio-therapy). They also mount responses to attempt to counteract danger signals and tissue stress; these include angiogenesis, remodeling of the ECM, and clearance of cellular debris [41]. Importantly, in mouse models of pancreatitis, they have been found to directly promote ADM via release of the pro-inflammatory cytokines RANTES and TNF, which activate NF- $\mathrm{B}$ in acinar cells and upregulate several ADM-related proteins such as MMP9 [42]. Macrophages also act in concert with $\mathrm{CD}^{+}{ }^{+} \mathrm{T}$ helper cells to direct adaptive immunity. Traditionally, they have been classified as M1 and M2 macrophages, depending on their mode of activation and cytokine profile, although this designation may be evolving. The imbalance in intratumoral macrophage-modulating factors usually leads to skewing of tumor-associated macrophages (TAMs) to an M2-like phenotype, as these tend to be pro-tumorigenic and T cell-suppressive [43]. By contrast, M1 macrophages usually support antitumor activity [43]. Since M1 polarization presumably correlates with the initiation of inflammatory responses, and M2 polarization with resolution, the perturbation observed in human and murine PDAC likely represents a maladaptive response with macrophages "locked" in an M2-like state that prevents antitumor immunity and supports tumor growth, invasion and metastasis as well as conferring resistance to chemoradiation [43-47]. An immunotherapeutic approach utilizing an agonistic CD40 antibody has shown promise in both 
murine and human PDAC through reprogramming of TAMs, which in turn became tumoricidal and facilitated depletion of tumor stroma [48]. TAM reprogramming was also achieved in another study in which mice with PDAC were subjected to inhibition of colony stimulating factor 1 (CSF1) or its receptor (CSF1R), resulting in reduced T cell-suppressive and augmented antigen presenting capacity [49].

$\mathrm{CD}^{+}$cytotoxic $\mathrm{T}$ lymphocytes (CTLs) are the main subset of antitumor effector cells; however they are usually relatively rare in PDAC-associated inflammatory infiltrates due to their exclusion by the associated stroma $[38,50,51]$. If present, they are suppressed by other immune and non-immune stromal cells via soluble factors such as IL-10 and TGF- $\beta$ and inhibitory ligands such as PD-L1 [49, 52] (Figures 4 and 5). Notably, the aberrant expression of PD-L1 on tumor cells requires the presence of inflammation, once again indicating that homeostasis is deranged in the presence of oncogenic mutations $[53,54]$. In addition, granulocytic and monocytic precursors of neutrophils and macrophages (and other myeloid cells), respectively, can give rise to subsets of myeloid-derived suppressor cells (MDSC), which, under the influence of tumor-derived factors such as GM-CSF, can directly inhibit antitumor immunity [31, 32, 55, 56]. Of note, most immunotherapeutic approaches aim to augment CTL effector responses.

$\mathrm{CD}^{+} \mathrm{T}$ cells have been reported to harbor either anti- or pro-tumorigenic effects, depending on their sub-differentiation. For instance, on the one hand, $\mathbf{T}$ helper $\mathbf{1}\left(\mathbf{T}_{\mathbf{H}} \mathbf{1}\right)$ cells have been shown to provide support to CTLs and to enable tumor rejection; however they are usually under-represented among intrapancreatic CD4 ${ }^{+} \mathrm{T}$ cells [57]. $\mathbf{T}_{\mathbf{H}} \mathbf{2}$ cells on the other hand, can promote pancreatitis and support pancreatic oncogenesis by enabling immune escape [17, 58, 59]. IL-17-producing CD4 ${ }^{+} \mathbf{T}$ cells $\left(\mathbf{T}_{\mathbf{H}} \mathbf{1 7}\right)$ are also present in the transformed pancreas and have been found to provide growth support to cancer cells overexpressing the IL-17 receptor 
under the influence of mutant Kras in a PDAC mouse model [60]. Lastly, regulatory $\mathbf{T}$ cells ( $\mathbf{T}_{\text {reg }}$ ), one of the most potent CTL-suppressive immune cell types, are recruited in the PDAC milieu via tumor-derived chemokines acting on the chemokine receptor CCR5 (such as CCL3 and CCL5), and their presence correlates with adverse patient outcomes [18, 28, 61]. Not surprisingly, ablation of $\mathrm{CD} 4^{+} \mathrm{T}$ cells in mice dramatically protects from pancreatitis and significantly delays PDAC development in the setting of mutant Kras [58, 59]. This exemplifies the inappropriate skewing of $\mathrm{T}$ helper cells in favor of pro-inflammatory and pro-tumorigenic subsets (i.e. $T_{H} 2, T_{H} 17$ and $T_{\text {reg }}$ ) in pancreatic cancer, constituting in turn, a typical example of failed defense mechanisms and deregulated homeostasis in the host.

An often neglected function of immune cells is their contribution to tissue regeneration. Accordingly, $\mathbf{R a g 1}^{1 /-}$ mice exhibit impaired pancreas regeneration following pancreatitis [62]. Although not well-studied in the context of pancreatic injury, IL-17 has an established role in the regeneration of other organs such as the liver [63]. It is likely that the aforementioned IL-17producing $\mathrm{T}$ cells are also part of a pro-regenerative program in response to bystander tissue injury in the pancreas; however the presence of oncogenic Kras mutations leads to a completely different outcome, as explained above [60]. Moreover, IL-22, a cytokine of the IL-10 family typically produced by $\mathrm{CD} 4^{+} \mathrm{T}$ cells (often together with IL-17), has been found to be secreted in response to Aryl hydrocarbon receptor (AhR) signaling and to promote tissue repair following acute pancreatitis in mice [64]. By contrast, chronic intraperitoneal administration of AhR ligands in mice with chronic pancreatitis has been shown to lead to high IL-22 production by $\mathrm{CD}^{+} \mathrm{T}$ cells, as well as PSC activation, in addition to exacerbated inflammation and fibrosis [65]. Interestingly, previous work examining Th17 cells in pancreatic oncogenesis also found elevated levels of IL-22 in PDAC-infiltrating $\mathrm{CD}^{+}{ }^{+} \mathrm{T}$ cells, suggesting that IL-22 may be 
contributing to PDAC fibro-inflammatory reaction and might constitute a link between smoking and other activators of AhR signaling, chronic pancreatitis, and PDAC [60].

Other immune cell types are also being identified to be co-opted by cancer cells and to contribute to pancreatic oncogenesis. B cells were recently found to infiltrate PDAC in both humans and mouse models $[66,67]$. Stromal cell-derived CXCL13 was identified as one of the main chemoattractants for B cells, which in turn promoted tumor progression directly, via secretion of IL-35, supporting cancer cell proliferation; and indirectly, via promotion of immunosuppressive TAMs and subsequent disabling of CTLs [66, 67]. Further, we have shown that $\gamma \delta \mathrm{T}$ cells infiltrate the chronically-inflamed pancreas, as well as early pre-neoplastic lesions and invasive PDAC in mice and humans; this in turn, prevents $\mathrm{CD} 4^{+}$and $\mathrm{CD} 8^{+} \alpha \beta$ T cells from mounting an antitumor immune response [52]. Mechanistically, the immunosuppressive effect of $\gamma \delta$ T cells was found to be mediated by T cell inhibitory ligands such as PD-L1 and Galectin-9 [52]. In mice, depletion of $\gamma \delta \mathrm{T}$ cells or deletion of chemokine receptors involved in their recruitment (e.g. CCR2, CCR5, or CCR6) led to a significant increase in the infiltration of CD4 ${ }^{+}$ and $\mathrm{CD} 8^{+} \alpha \beta \mathrm{T}$ cells, and afforded protection from the development and progression of PDAC lesions [52]. Of note, $\gamma \delta \mathrm{T}$ cells have also been reported to be an intrapancreatic source of IL-17; however their pro-tumorigenic effects appear to be independent of IL-17 production since IL-17 levels are not significantly affected upon $\gamma \delta \mathrm{T}$ cell depletion $[52,60]$. In these human correlative studies, the majority of intrapancreatic $\gamma \delta$ T cells were found to be $\mathbf{V} \boldsymbol{\delta} \mathbf{1}^{+}$, and this particular subset has been previously reported to have a protumorigenic role; by contrast, $\mathrm{V} \delta 2^{+} \mathrm{T}$ cells harbor tumoricidal capacity and have been exploited for immunotherapy against PDAC and other malignancies [68]. 


\section{Cancer-associated Fibrosis}

Tissue fibrosis has its roots in the normal wound healing process, particularly at sites where adequate regeneration cannot occur. Resident tissue fibroblasts are normally activated by acute inflammation and engage in functions that promote epithelial regeneration, including angiogenesis, modulation of the inflammatory response, provision of growth signals, as well as synthesis of ECM proteins that can act as a cellular scaffold for epithelial cells, among other things [10]. Unlike the physiologic state where fibroblasts either revert back to quiescence or die once tissue integrity is restored, chronic inflammatory states (such as chronic pancreatitis and PDAC) are characterized by persistent activation of fibroblasts and other stromal cells, leading to the formation of an abnormal ECM that contributes to a state of desmoplasia.

Although desmoplasia was identified as a hallmark of PDAC several decades ago, its pathophysiologic significance is not well understood $[69,70]$. The predominant effector cell is the CAF, which originates from activated resident fibroblasts (such as pancreatic stellate cells). CAFs exhibit bi-directional communication with both epithelial cells and immune cells $[38,71]$. They are activated by DAMPs, cytokines, and various other agents (including chemotherapeutics) and contribute to PDAC progression through a myriad of mechanisms (Fig. 5). Although attempts to deplete CAFs have yielded contradictory results, they remain a very attractive target for therapeutic intervention since they represent a central hub coordinating aberrant stromal homeostasis [69, 72-74].

The fibro-inflammatory response of PDAC is visible very early on in the process of carcinogenesis, even at the stage of ADM, and is then progressively intensified [69]. The end result is the generation of a dense, gel-like extracellular matrix with unique physicochemical properties [75]. Several macromolecules of the gel-like ECM, such as hyaluronic acid (HA), 
absorb and retain significant amounts of water, thus increasing the interstitial fluid pressure (IFP) of the tumor; this dramatically elevated IFP is evident by the collapsed blood vessels in areas of desmoplasia, and appears to be an important hurdle to adequate delivery of drugs in the tumor milieu, due to both inadequate perfusion and opposition to their extravasation [75].

Interestingly, similar stromal modifications have been found not only in primary tumors, but also at sites of future metastases. For example, in both murine and human PDAC, the metastatic liver has been shown to exhibit significant alterations in its microarchitecture that are evident even with isolated tumor cells [70, 76]; these include the deposition of various ECM proteins (such as HA, collagen I and fibronectin), and activation of myofibroblast-like cells, as well as infiltrating immune cells $[70,76]$. These changes are remarkable in that they have been shown to promote the engraftment of pancreatic cancer cells in mouse models and thus, constitute a defining characteristic of the hepatic premetastatic niche [77, 78]. Successful treatment of liver metastases in mice leads to regression of metastasis-associated ECM changes, attesting to the addiction of pancreatic cancer cells to desmoplasia [76].

\section{Additional Local and Systemic Imbalances}

\section{Autophagy, Endoplasmic Reticulum Stress, and Metabolic Perturbations}

Stressed pancreatic epithelial cells accumulate metabolic intermediates that damage vital macromolecules and intracellular organelles. One of their cardinal defense mechanisms is the process of autophagy, which allows the removal of cellular debris that propagate cellular injury; limits the accumulation of DAMPs and the resultant inflammatory reaction; and recycles essential metabolic intermediates to facilitate cellular repair (reviewed in [12]). Disruption of autophagy via genetic deletion of its key mediator autophagy related 5 (ATG5) in mice led to 
increased levels of reactive oxygen species, augmented endoplasmic reticulum (ER) stress, and development of spontaneous chronic atrophic pancreatitis [79]. Conceivably, autophagy appears to limit ER stress and pro-tumorigenic mediators, and protect during incipient pancreatic oncogenesis $[80,81]$.

The coin is flipped in established PDAC: both mutant Kras and microenvironmental stressors, such as intratumoral hypoxia and nutrient deprivation, significantly increase autophagic flux, which benefits cancer cells by protecting them from toxic intermediates, including chemotherapeutics, and by supporting tumor metabolism [82, 83]. Cancer cells have a very high turnover of proteins, lipids, and other molecules, and therefore rely on a "personalized menu" of metabolic intermediates, as well as on raw material derived through autophagy [12, 15]. Metabolic reprogramming is considered a hallmark of cancer, and likely originates from homeostatic circuits that attempt to mobilize readily usable intermediates to support stressed cells [83]. However, its effects not only nurture the cancer cells but also affect the TME and propagate tumor-enabling inflammation. Further, epithelial and stromal cell crosstalk leads to changes in the metabolic intermediates in the tumor milieu that support the cancer cells [84]. One study recently showed that human cancer cells can coerce PSC to activate autophagy in vitro so as to provide non-essential amino acids such as alanine that fuel the cancer cells' tricarboxylic acid cycle and support lipid and protein synthesis [85]. The above were verified in vivo using xenograft mouse models, although the cancer cell-derived mediators responsible for this process remain unidentified.

Conditions that further deregulate metabolism, such as obesity, can also fuel inflammation and accelerate pancreatic carcinogenesis [86]. For example, a recent study in an orthotopic PDAC mouse model found that obesity leads to adipocyte activation, which can in 
turn recruit neutrophils to the pancreas via IL-1 $\beta$ signaling [87]. The latter can then enhance PSC activation, thus supporting tumor growth and augmented desmoplasia, promoting chemoresistance [87]. Adipocytes have also been shown to promote proliferation of murine pancreatic cancer cells in vitro by providing glutamine, especially in nutrient-poor conditions [88]. On the other extreme of metabolic perturbations is cancer cachexia, a dreaded complication of PDAC which leads to wasting, and occurs with advanced disease. In mouse models of PDACassociated cachexia, several tumor-derived pro-inflammatory factors (e.g. IL-6 and TGF- $\beta$ ) have been shown to modulate the metabolism at a systemic level, as evidenced by altered levels of circulating glucose and ketones, $[89,90]$. The metabolic stress leads to elevated glucocorticoid levels that contribute to immune suppression and tumor tolerance [89].

In summary, it is now becoming evident that several homeostatic processes that were traditionally viewed at the cancer cell level, such as the response to cellular stress and the regulation of metabolism, need to be revisited and considered in conjunction with the tumor's surrounding stromal cells.

\section{Coagulation}

Another consequence of deregulated homeostasis at a systemic level is the unbalanced activation of the coagulation system. Thromboembolic disease occurs in up to $57 \%$ of PDAC patients and in extreme cases it may manifest as migratory thrombophlebitis (Trousseau's syndrome) or disseminated intravascular coagulation [91, 92]. Although initially theorized to be related to tumor cell emboli and mucins secreted by tumor cells into the circulation, the hypercoagulability associated with cancer is now believed to be a result of multiple overlapping mechanisms involving imbalances in positive and negative regulators of the coagulation 
pathway; secretory products of cancer cells and activated immune cells; systemic changes in endothelial cells of both venous and arterial conduits; as well as widespread platelet activation [92]. Importantly, several intermediates of the coagulation cascade including fibrinogen may act as mitogenic signals for fibroblasts that perpetuate chronic inflammation [93, 94].

\section{Metastasis}

An important evolving phenomenon in our understanding of metastasis is the contribution of the immune system to this process [76, 95]. Metastasis has been conceptualized as a sequential process whereby cancer cells detach from their surroundings, migrate through the extracellular matrix, gain access to blood vessels or lymphatics, travel to distant sites, and exit these conduits at distant organs, remaining dormant or forming metastatic foci [96]. The immune system has been shown to contribute to all these steps through multiple complementary mechanisms (reviewed in [95]). In PDAC, one recent study showed that macrophages were recruited to the liver of mice at sites seeded by isolated cancer cells [47]. There, they secreted granulin, a factor that in turn activated hepatic stellate cells to synthesize a dense ECM, and supported tumor cell proliferation in the liver in a periostin-dependent manner, thus promoting metastasis. The above is reminiscent of a foreign body reaction, or a granuloma formation in response to infectious agents; however, a main difference here is that cancer cells can exploit it to their advantage.

Even more strikingly, deranged immune responses can create premetastatic niches altered microenvironments in distant organs that are conducive to future colonization by circulating tumor cells. For example, in an orthotopic mouse model of PDAC as well as in intraperitoneally disseminated colon cancer mouse model, tumor cells were found to promote the 
expansion of intrahepatic MDSC through the release of soluble factors such as KC/CXCL1, creating an immune-privileged hepatic microenvironment that favors [97]. Moreover, Kruger's laboratory recently showed that stromal cells in human and murine PDAC and other tumors could release tissue inhibitor of metalloproteinases-1 (TIMP-1), which acts in the premetastatic liver to induce the secretion of ECM proteins such as fibronectin, and chemokines such CXCL12 $[98,99]$; the interaction of TIMP-1 with CD63 on hepatic stellate cells mediates some of these effects and leads to neutrophil recruitment in a CXCL12/CXCR4-dependent manner, supporting liver homing of cancer cells $[98,99]$. Another study reported that murine PDAC-derived exosomes constitute an important source of immunomodulating factors such as macrophage migration inhibitory factor (MIF); these are taken up by hepatic Kupffer cells to induce a TGF$\beta$-mediated signaling cascade that involves ECM remodeling and recruitment of additional bone marrow-derived cells, ultimately promoting murine PDAC metastasis to the liver [77].

Collectively, these current findings exemplify how primary tumors can globally affect other biological processes at an organismal level that allow them to survive hostile microenvironments, evade destruction by the immune system, grow and spread to distant organs. Additional mechanisms that are likely to underlie PDAC metastasis include the modulation of distant organ microvessel architecture (e.g. in response to DAMPs and low-grade inflammation) to facilitate disseminated tumor cell (DTC) homing and extravasation; modulation of metabolism to support DTC growth; and altered angiogenesis in response to hypoxia, as has been shown for other cancer types [100].

\section{Concluding Remarks}


It is now clear that the tumor microenvironment is of vital importance in influencing the behavior of tumor cells, their ability to evolve and spread, as well as their response to chemoimmune-therapeutic treatments. It must be appreciated that the relationship between deregulated homeostatic responses and cancer progression is most likely not a deterministic phenomenon. Instead, it is a consequence of natural selection whereby cancer cells that are able to thrive in conditions of chronic inflammation and fibrosis are selectively, the ones that survive. The presence of multiple clones of cancer cells further complicates this picture, as these malignant clones might exploit host physiology by benefiting from chronic inflammation in different ways and/or using redundant pathways. As a result, devising anti-cancer therapies to attack a single physiological process is frequently ineffective. Several novel discoveries in PDAC have revealed new possibilities but have also generated several questions that need to be answered (see Outstanding Questions). By thinking of the pathophysiology of the tumor microenvironment as a whole and in the context of deranged homeostatic responses, me may better understand the process of cancer development and its resistance to treatment, which may inform novel ways to profile (Box 1) and treat such a devastating disease (Box 2). As an analogy, one might consider the management of septic shock, where antibiotics alone are not enough to treat it, given that an important part of its pathophysiology originates from a derailed immune response; thus, approaches to treating pancreatic cancer may be viewed in a parallel fashion, where it is important to consider the tumor in relation to the host's physiology, and vice versa. Outcomes may improve only when interventions targeting the perturbed immune homeostasis are instituted: it is time to think outside the box! 


\section{Acknowledgments}

CPZ is supported by the Center for Metastasis Research Fellowship (Memorial Sloan Kettering Cancer Center, NY). GM is supported by NIH grants CA168611, CA155649, and CA206105; the Department of Defense Peer Reviewed Medical Research Program; the Lustgarten Foundation; AACR-PanCan; and the Hirshberg Foundation for Pancreatic Cancer Research.

\section{Conflict of Interest}

None declared. 


\section{Figure Legends}

\section{Key Figure, Figure 1. Homeostatic Responses to Tissue Stress in the Context of Pancreatic}

\section{Dysfunction and Malignancy.}

Cellular stress can be invoked by a great variety of internal (e.g. wear and tear/aging, reactive oxygen species, hypoxia, etc) and external causes (e.g. toxins, radiation, infectious agents, trauma, etc). Some can be mutagenic, potentially leading to cancer. Stressed/damaged cells activate defensive systems that attempt to repair the injury. If successful, the cell returns to its normal state and usually minimal or no inflammatory response is induced (a).

(b-d) If the injury is extensive and irreparable, the cell may either go into a senescence - a "safe mode" characterized by cell cycle arrest and slowing of cellular functions [101] - or may activate programmed cell death by apoptosis or necroptosis. If the injury is sudden and overwhelming, it may induce cell rupture and resultant necrosis. Both senescence and the various modes of cell death are characterized by altered expression of cell surface molecules as well as release of soluble mediators that activate the immune system [101, 102]. The resultant inflammatory response attempts to clear off the debris and support tissue regeneration. If the normal homeostatic mechanisms malfunction, the inflammation may persist leading to a futile cycle of further tissue injury, fibrosis, and potentially carcinogenesis.

(e) Malignant transformation usually arises de novo secondary to serial mutagenic insults to normal cells. The immune system usually recognizes single transformed cells and clears them off (elimination). Occasionally, some transformed cells may go unrecognized by the immune system and persist. As they accumulate additional mutations, they may initiate an inflammatory response that can clear them off. At the same time though, the inflammatory response places a selection pressure on the transformed cells which may enable the emergence of "resistant" clones and 
eventual escape from immunosurveillance mechanisms [103]. One of the ways the immune system escapes is by maintaining an immune infiltrate permissive to tumor growth through release of pro-tumorigenic mediators and simultaneous exclusion of cytotoxic cells (see Fig. 4). Finally, the sustained intratumoral inflammatory response may induce collateral damage to surrounding non-transformed epithelial cells. Activation of the responses to cell injury described above leads to release of additional pro-inflammatory mediators and further perpetuation of tumor-associated inflammation.

\section{Figure 2. Simplified Homeostatic Circuit of the Inflammatory Response to Tissue Stress.}

(a) The general composition of a homeostatic circuit (examples include control of blood glucose levels, tissue oxygenation, and core body temperature). The set point may be fixed at a certain range (which may vary from between tissues or organs), or may be adjustable depending on the context (e.g. the body temperature set point is raised during the febrile response). The sensitivity (or gain) of the sensors can be fine-tuned, making them more or less responsive to deviations from the set point.

(b) Inflammation has evolved to protect the organism from intrinsic and extrinsic challenges that, if not contained, can be detrimental to the involved tissue or even the entire body. It usually obeys the laws of homeostasis (although the system is in reality, more complex). Activators of the immune system (inducers, such as those described in Key Figure, Figure 1) are identified by the sensors as deviations from the set point, as a result of altered cell surface molecules and release of soluble mediators. The sensors initiate downstream cascades that attempt to alleviate the initial cause that deranged the system. In several disease states characterized by chronic inflammation (such as pancreatic cancer), the system is derailed secondary to imbalances 
between positive and negative regulators (e.g. additional activating signals elicited by cancer cells). These imbalances create new set points, modulate the threshold for sensor activation (gain) and alter the functions of effectors, such that instead of returning to baseline (i.e. homeostasis) the system is trapped in a self-perpetuating infinite loop that only benefits the cancer cells.

\section{Figure 3. Examples of Regulated and Dysregulated Inflammatory Reactions in the}

\section{Pancreas.}

The output of a homeostatic system (e.g. degree of inflammation in the pancreas) can be plotted in a graph against time (top). In healthy tissues, pro-inflammatory mediators and downstream immune responses fluctuate within a narrow "normal range" that are not sufficient to trigger inflammation at the steady state. Cellular stress or injury can trigger an inflammatory reaction of variable magnitude, which can range from a minimal reaction to tissue stress (parainflammation); to a more pronounced inflammatory reaction to overt cellular injury, as in mild acute pancreatitis (AP), characterized by local inflammation; to a full-blown systemic inflammatory response as seen in severe AP. The inflammatory response is driven by positive regulators (e.g. IL-6), but is eventually tuned down by negative regulators of the system (e.g. IL10; bottom graph). Therefore, inflammation eventually subsides and the system returns back to normal (homeostasis).

When Kras is mutated, this balance is disturbed in two ways: (i) Kras mutant cells have a lower threshold for release of pro-inflammatory mediators; and (ii) the balance of positive and negative regulators of inflammation is tilted towards one that favors a sustained activation of immunosuppressive and pro-tumorigenic subsets. 
Besides pro-inflammatory signals from transformed cells, the tumor-enabling inflammation is further perpetuated by (i) soluble mediators released by stressed non-transformed epithelial cells within the tumor microenvironment; and (ii) systemic promoters of low-grade inflammatory states such as smoking, obesity, and microbial dysbiosis.

\section{Figure 4. Mediators of Tumor-Enabling Inflammation within the Pancreatic Cancer}

\section{Microenvironment.}

Cancer cells secrete a vast array of soluble factors that support an inflammatory infiltrate permissive of tumor growth. This is further fueled by similar mediators supplied by stressed nontransformed epithelial cells, particularly DAMPs that engage pattern recognition receptors (e.g. TLR-4, -7, and-9; CLR such as Mincle and Dectin-1; RAGE, etc) The effects of each mediator are far more complex than shown above. Several of them can be secreted by more than one source and act on multiple cell types. For example TGF- $\beta$ and IL-10 can be secreted by both the cancer cells and regulatory T cells (Treg). TGF- $\beta$ can supports Treg differentiation as well as $\mathrm{T}_{\mathrm{H}} 2$ skewing of $\mathrm{CD}^{+} \mathrm{T}$ cells and disabling of $\mathrm{CD} 8^{+} \mathrm{CTLs}$. The combination and relative ratios of different soluble mediators will ultimately dictate the outcome. Furthermore, cell surface molecules expressed on tumor and stromal cells (such as PD-L1) can also disable CTLs.

The end results of tumor-enabling inflammation can be conceptualized as (i) provision of cancer cells with growth signals (e.g. IL-6, IL-17); (ii) suppression of CTL anti-tumor activity; and (iii) activation of other non-immune stromal cells (shown in Figure 5).

HMGB1, high mobility group box 1; SAP130, Sin3-associated protein 130; TAN, tumorassociated neutrophils. 


\section{Figure 5. Dysregulated Fibroblast Function in PDAC Leads to Desmoplasia where Cancer}

\section{Cells Thrive.}

CAFs have a bidirectional communication with the epithelial compartment of the tumor.

Epithelial-derived factors such as cytokines and chemokines, as well as DAMPs that ligate PRRs

promote their activation via the MAPK, NF- $\kappa \mathrm{B}$, and STAT3 pathways $[17,18,71,104]$. CAFs in turn provide, pro-proliferative and anti-apoptotic signals [18, 71, 105, 106]; promote angiogenesis [71, 104, 107]; modulate the ECM; and contribute to chemo- and radio-resistance $[105,106]$. Importantly, CAFs also engage in crosstalk with immune cells and promote tumor tolerance: they recruit immunosuppressive cells such as Treg and MDSC $[18,104]$; they skew helper $\mathrm{T}$ cells to $\mathrm{T}_{\mathrm{H}} 2$ deviation $[25,107,108]$; they sequester cytotoxic $\mathrm{CD}^{+} \mathrm{T}$ cells away from cancer cells $[38,51]$; and recruit neutrophils to distant organs such as the liver, generating premetastatic niches $[98,99]$. A newly appreciated mode of intercellular communication is exosomes, which are upregulated on CAFs under stressful conditions, such as hypoxia and exposure to chemotherapy [106, 109]. All the aforementioned mechanisms make the TME more hospitable to cancer cells, and contribute to tumor growth, immune escape, and metastasis. Agents such as all-trans retinoic acid (ATRA) may have a role in blocking their activation and disrupting their pro-tumorigenic function [110].

IFP, interstitial fluid pressure; MMP, matrix metalloprotease; OPN, osteoprotegerin; TSLP; thymic stromal lymphopoietin; VEGF, vascular endothelial growth factor. 


\section{Boxes}

\section{Box 1. Tumor Profiling Should Include the Microenvironment.}

The traditional approach to most solid malignancies has been surgical removal of the tumors when feasible, accompanied by high-dose chemotherapeutic agents postoperatively, depending on clinicopathological parameters that dictate the risk of locoregional and or distant recurrence. If resection is not feasible due to size or involvement of critical surrounding structures (e.g. major blood vessels), the patient may be treated with chemotherapy and/or radiation therapy first, in an attempt to "shrink" the tumor and convert it to resectable. If the tumor is unresectable to begin with, the patient is usually treated with high doses of chemotherapy. Even though most of these treatments have the potential to prolong survival, they often come with serious side effects that compromise a patient's quality of life. Ultimately, the tumors recur and metastatic disease with its associated complications/death ensues.

This remarkable ability of cancer to recur originates from is its inherent potential to mutate and adapt to its environment. The selection pressure imposed by chemotherapeutic agents will promote the emergence of a minority of resistant clones that may find shelter under the protective veil of the co-opted fibro-inflammatory stroma. They remain dormant until they gain strength and eventually form new colonies. This phenomenon of resistance is not entirely cellautonomous, but it also depends on the microenvironment that may supply a favorable scaffold, soluble mediators, nutrients, and signals that shield the cancer cell from chemotherapeutic agents.

In the past decade, several novel types of biomarkers have been introduced to clinical practice that can either enhance the accuracy of prognosis or predict response to certain types of adjuvant therapies, and have gained clinical utility in breast cancer. Although useful, such 
approaches of mutational analysis entirely neglect the stroma and the systemic perturbations of homeostatic responses. Not surprisingly, they haven't been successful in PDAC, which relies so heavily on fibro-inflammation. We therefore need to capture the status of the tumor microenvironment in order to better identify opportunities for treatment.

A theoretical example to better profiling would be a combination of:

- Histopathological and immunohistochemical analysis of tumor and peritumoral tissue (reaction to tumor), including immune infiltrates, lymphovascular and neural structures, fibrosis etc

- Mutational analysis of cancer cells

- Microdissection of tumor stroma and subsequent gene expression profiling

- Analysis of upregulated circulating cytokines, chemokines

- Circulating exosome profiling

- Intestinal microbiome profiling

- Analysis of a host's gene polymorphisms that may be potentially implicated in homeostatic response outcomes and pathophysiology.

\section{Box 2. Clinician's Corner: A Novel Approach to Cancer Treatment}

Imagine if we could gather extensive intelligence (comprehensive profiling, such as the theoretical example in Box 1) before attacking the enemy (the cancer cells). Once we know what is happening behind the enemy lines, we first take down the enemy's supply line, destroy the supportive infrastructure, disable the defenses, and isolate the enemy before mounting our headson attack. The enemy will have nowhere to hide and would be entirely vulnerable to our weapons (e.g. chemotherapy). What if we could approach cancer in this way, i.e. restore the 
aberrant homeostatic responses back to normal (to the extent that it is possible) and then deal with the cancer cells, while continuing to prevent aberrant physiology. With appropriate intelligence we could also predict the stromal responses after treatment and manage them preemptively (e.g. by depleting DAMPs, or using neutralizing antibodies for chemokines or TLRs).

Ultimately, the goal should be not only to eradicate the transformed cells, but also to restore the lost equilibria and re-establish homeostasis. To this end, it must be kept in mind that non-targeted therapies such as traditional chemotherapeutics and radiation therapy can injure the uninvolved parenchymal cells and the surrounding supportive tissue - particularly when delivered at maximal tolerated doses - thus propagating the vicious cycle of inflammation and fibrosis. On the contrary, lowering the treatment doses and combining multiple drugs with targeted agents and other modalities might be the key to delivering a more accurate hit to cancer cells while sparing the stroma and uninvolved epithelial cells. This is an area that certainly deserves further exploration.

\section{Glossary}

- Acinar-to-ductal metaplasia (ADM): process of de-differentiation of pancreatic acinar cells and assumption of immature ductal epithelial cell features in response to cellular stress and injury. It is necessary for regeneration and is reversible once the noxious stimulus is removed; however in the presence of Kras mutations it becomes irreversible and can proceed to PanIN formation and PDAC development.

- Angiogenesis: the process of forming new blood vessels. It occurs physiologically during development, as well as transiently during wound healing in adults. Cancers cells release various factors that stimulate angiogenesis in a persistent and haphazard fashion, in order 
to augment oxygen and nutrient supply and sustain their heightened proliferation and metabolism.

- Aryl hydrocarbon receptor (AhR): a cytosolic ligand-activated transcription factor with important roles in $\mathrm{T}$ cell differentiation and xenobiotic response to toxic metabolites. It is activated by binding various naturally occurring compounds as well as synthetic polycyclic aromatic hydrocarbons and various other toxic intermediates.

- Autophagy: the process of recycling damaged intracellular organelles and macromolecular structures via sequestration in double-membrane vesicles (termed 'autophagosomes') that eventually fuse with lysosomes leading to degradation of their contents. It is upregulated under conditions of starvation as a homeostatic response to nutrient deprivation.

- Bystander effects of homeostatic responses: whenever a tissue is exposed to stress (e.g. radiation injury), it activates protective responses to shield it from the injurious insult. In the case of cancer, malignant cells may benefit from mediators (e.g. chemokines) released by non-malignannt cells as part of these protective responses.

- Cachexia: A multifactorial clinical syndrome associated with advanced malignancy, characterized by weight loss, skeletal muscle wasting, poor performance status, and partial refractoriness to nutritional supplementation. It is especially prominent in PDAC and correlates with decreased survival.

- Cancer-associated fibroblasts (CAFs): mesenchymal cells originating from tissueresident fibroblasts that, under the influence of tumor-derived cues, assume an activated secretory phenotype providing nutrients and growth signals to cancer cells; supporting the propagation of cancer-associated inflammation; and contributing to resistance to 
chemotherapy, radiation therapy and immunotherapy. There is no universal specific marker for their detection, although CD140a (PDGF-R $\alpha$ ) and fibroblast activation protein (FAP) have been proposed in separate studies.

- Coagulation cascade: The main defense system responsible for hemostasis. Upon exposure of subendothelial collagen, tissue factor, and other molecules (e.g. due to endothelial injury), platelets adhere to the site and become activated, initiating the sequential activation of coagulation factors. The end result is the generation of a fibrin polymer that stabilizes the adherent platelets and contributes to the inflammatory response.

- Complement system: A set of soluble factors that continuously probe for invading pathogens as well as damaged cells and supplement the immune system's function in eliminating the former. It is activated rapidly and results in generation of multiple intermediates that contribute not only to immune clearance but also to inflammation, angiogenesis, tissue repair, as well as various pathogenic conditions.

- Damage-associated molecular patterns (DAMPs): molecules released by injured or dying cells (e.g. S100 proteins, heat shock proteins, HMGB1, fibronectin, etc), which bind to and activate pattern-recognition receptors (e.g. Toll-like receptors).

- Desmoplasia [GR: $\delta \varepsilon \dot{\varepsilon} \sigma \mu \eta$ (bundle) $+\pi \lambda \dot{\alpha} \theta \omega$ (to make)]: the intense fibrotic reaction that accompanies neoplastic and non-neoplastic processes and is result of deranged remodeling of the ECM.

- Extracellular matrix (ECM): a dense, complex substance that fills the interstitial space of organs. It is composed of various fibrillary (such as collagen) and globular proteins, as well as glycosaminoglycans and proteoglycans - the main one being hyaluronic acid 
(HA). It functions as a supportive mesh for epithelial cells, providing contact signals via cell surface molecules (e.g. integrins). It is usually formed by fibroblasts and serves as a reservoir for growth factors (e.g. TGF- $\beta$ ), chemokines and other molecules which are embedded in the matrix and are released during the remodeling phase.

- Exosomes: microvesicles (40-150 $\mathrm{nm}$ in diameter) originating from inward budding of the plasma membrane and subsequent processing in multivesicular bodies, with eventual release in the surroundings; they carry proteins and nucleic acids (mRNA, miRNA, DNA) and are emerging as key players in intercellular communication in health and disease.

- Gamma delta $(\gamma \delta) \mathrm{T}$ cells: $\mathrm{T}$ cells bearing the $\gamma \delta \mathrm{T}$ cell receptor (TCR). They are not antigen-restricted and recognize antigens on pathogens as well on stressed cells via their TCRs and other receptors (e.g. PRRs). They are found in the peripheral blood as well as in various organs that interface with the external environment (e.g. skin, IEL compartment, liver etc) and are among the first responders to tissue stress and infection. Recent studies have found conflicting roles for $\gamma \delta$ T cells in carcinogenesis. Based on the $\delta$ chain of their TCR, two main subsets have been identified in humans - V反 1 and $\mathbf{V} \boldsymbol{\delta} 2$, although $\mathrm{V} \delta 3$ and other much less studied subsets exist.

- Granulin: glycoprotein involved in wound healing. It is associated with fibroblast migration and has variable effects on epithelial cell growth.

- Intraepithelial lymphocytes (IELs): immune cells of lymphocyte lineage that reside within the epithelial lining of the mucosa of various organs (such as the intestine). Unlike naïve $\mathrm{T}$ cells (that have not been previously exposed to antigens), they do not require 
priming and are able to be activated rapidly, thus serving as a first line of defense against invading pathogens as well as stressed epithelial cells.

- Kupffer cells: liver-resident macrophages located within the lumen of hepatic sinusoids, adherent to their walls, and having important roles in sampling and clearance of damaged cells and microbes in health and disease. Upon activation, they release several soluble mediators that can affect immune responses locally within the liver as well as systemically (e.g. in the bone marrow).

- M1 and M2 macrophages: conceptual framework of macrophage polarization, initially described for infectious diseases and imperfectly applied to tumor-associated macrophages. M1 ('classical') are induced by interferon- $\gamma$ and LPS and promote $\mathrm{T}_{\mathrm{H}} 1$ responses, while M2 ('alternative') macrophages are induced by IL-4, IL-10, IL-13, and FcR $\gamma$ signaling and support $\mathrm{T}_{\mathrm{H}} 2$ responses.

- Metabolic reprogramming: alterations in the cancer cell's metabolism in response to cellular stress, increased proliferation and hypovascularity that lead to relative nutrient deprivation and hypoxia. A classic example is the augmented uptake of glucose and preferential funneling into glycolysis, even under normoxic conditions, termed the 'Warburg effect' after Otto Warburg.

- Necroptosis: a recently identified process of cell death with morphological features of necrosis but a more organized mode of initiation and propagation (resembling apoptosis), via engagement of death receptors or several PRRs, and activation of receptor interaction protein kinase 1 (RIP1) and RIP3 downstream : 'programmed necrosis'.

- Opsonization: the process of coating a cell (endogenous, such as apoptotic cell; or exogenous, such as a microbe) with "opsonins" - molecules that promote complement 
activation on the surface of that cell. Such molecules include antibodies (especially $\operatorname{IgM}$ ), complement proteins (e.g. C3b), and other circulating proteins (e.g. ficolins).

- Pancreatic Intraepithelial Neoplasia (PanIN): premalignant alterations of the pancreatic ductal epithelium. According to the overall cellular morphology as well as the nuclear architecture and atypia, they are subdivided into PanIN-1, -2 , and -3 - the latter representing carcinoma in situ. They correlate with progressive accumulation of mutations and increasing risk for progression to PDAC. PanIN-1 and -2 are quite common in patients over 40 years.

- Pancreatic stellate cells (PSCs): fibroblast-like cells that reside in the pancreas in a quiescent form, characterized by expression desmin and storage of vitamin A. When activated (e.g. by cellular injury/stress, cytokines, chemokines, DAMPs), they assume a myofibroblast phenotype, lose vitamin A cytoplasmic droplets, and express $\alpha$-smooth muscle actin ( $\alpha$-SMA). They are the primary source of ECM molecules in health and disease, and contribute to pancreatic fibrosis, regeneration, inflammation and tumorigenesis. They are likely the primary source of CAFs in the context of pancreatic cancer although this is an area of ongoing investigation and debate.

- Pattern recognition receptors (PRRs): a broad class of receptors important in the innate immune system. They sense evolutionarily conserved molecular motifs originating from microbes (e.g. lipopolysaccharide, LPS) as well as from stressed cells (DAMPs) and contribute to various immune-related processes. They comprise four families: Toll-like receptors (TLRs); C-type lectin receptors (CLRs; e.g. Dectin-1); NOD-like receptors (NLRs); and RIG-like receptors (RLRs). 
- Periostin: ECM protein normally found in periosteum of bones (hence the name). It is upregulated in several cancer types (e.g. ovarian and pancreatic cancer) and interacts with multiple receptors, such as integrins, on cancer cells to facilitate their survival, invasion, and metastasis, among others.

- $\operatorname{Rag} 1^{-/-}$mice: genetically-engineered mice harboring an inactivating mutation of the recombination activation gene $\operatorname{Rag}-1$, resulting in defective $\mathrm{V}(\mathrm{D}) \mathrm{J}$ recombination of $\mathrm{B}$ and $\mathrm{T}$ cell receptors and hence lack of $\mathrm{B}$ and $\mathrm{T}$ cells due to early developmental arrest.

- Schwann cells: glial cells that are wrapped around nerve fibers of peripheral nerves. They synthesize the insulating myelin sheath and provide structural support to peripheral neurons.

- Stroma: the supportive (i.e. non-parenchymal) part of an organ. It can be divided into an acellular component (the ECM) and a cellular component. The latter is composed of a variety of cells such as mesenchymal stem cells, fibroblasts, vascular and lymphatic endothelial cells, neurons and Schwann cells (or other nerve supportive cells), adipocytes, etc. 


\section{References}

1 Greer, J.B. and Whitcomb, D.C. (2009) Inflammation and pancreatic cancer: an evidencebased review. Curr. Opin. Pharmacol. 9, 411-418

2 Siegel, R.L., et al. (2017) Cancer Statistics, 2017. CA Cancer J. Clin. 67, 7-30

3 Ricklin, D., et al. (2010) Complement: a key system for immune surveillance and homeostasis. Nat. Immunol. 11, 785-797

4 Kotas, M.E. and Medzhitov, R. (2015) Homeostasis, inflammation, and disease susceptibility. Cell 160, 816-827

5 Chen, G.Y. and Nunez, G. (2010) Sterile inflammation: sensing and reacting to damage. Nat. Rev. Immunol. 10, 826-837

6 Takeuchi, O. and Akira, S. (2010) Pattern recognition receptors and inflammation. Cell 140, $805-820$

7 Cao, X. (2016) Self-regulation and cross-regulation of pattern-recognition receptor signalling in health and disease. Nat. Rev. Immunol. 16, 35-50

8 Iwasaki, A. and Medzhitov, R. (2015) Control of adaptive immunity by the innate immune system. Nat. Immunol. 16, 343-353

9 Dvorak , H.F. (1986) Tumors: Wounds That Do Not Heal. N. Engl. J. Med. 315, 1650-1659

10 Dvorak, H.F. (2015) Tumors: wounds that do not heal-redux. Cancer Immunol Res 3, 1-11

11 Zheng, L., et al. (2013) Role of immune cells and immune-based therapies in pancreatitis and pancreatic ductal adenocarcinoma. Gastroenterology 144, 1230-1240

12 Gukovsky, I., et al. (2013) Inflammation, autophagy, and obesity: common features in the pathogenesis of pancreatitis and pancreatic cancer. Gastroenterology 144, 1199-1209 e1194 13 Zambirinis, C.P., et al. (2014) Pancreatic cancer, inflammation, and microbiome. Cancer J. 20, 195-202

14 Yeo, T.P. and Lowenfels, A.B. (2012) Demographics and epidemiology of pancreatic cancer. Cancer J. 18, 477-484

15 Kleeff, J., et al. (2016) Pancreatic cancer. Nat Rev Dis Primers 2, 16022

16 di Magliano, M.P. and Logsdon, C.D. (2013) Roles for KRAS in pancreatic tumor development and progression. Gastroenterology 144, 1220-1229

17 Ochi, A., et al. (2012) MyD88 inhibition amplifies dendritic cell capacity to promote pancreatic carcinogenesis via Th2 cells. The Journal of experimental medicine 209, 1671-1687

18 Zambirinis, C.P., et al. (2015) TLR9 ligation in pancreatic stellate cells promotes tumorigenesis. J. Exp. Med. 212, 2077-2094

19 Seifert, L., et al. (2016) The necrosome promotes pancreatic oncogenesis via CXCL1 and Mincle-induced immune suppression. Nature 532, 245-249

$20 \mathrm{Kang}$, R., et al. (2010) The receptor for advanced glycation end products (RAGE) sustains autophagy and limits apoptosis, promoting pancreatic tumor cell survival. Cell Death Differ. 17, 666-676

21 Ochi, A., et al. (2012) Toll-like receptor 7 regulates pancreatic carcinogenesis in mice and humans. J. Clin. Invest. 122, 4118-4129

22 Farrell, J.J., et al. (2012) Variations of oral microbiota are associated with pancreatic diseases including pancreatic cancer. Gut 61, 582-588

23 Fan, X., et al. (2016) Human oral microbiome and prospective risk for pancreatic cancer: a population-based nested case-control study. Gut 
24 Mitsuhashi, K., et al. (2015) Association of Fusobacterium species in pancreatic cancer tissues with molecular features and prognosis. Oncotarget 6, 7209-7220

25 De Monte, L., et al. (2011) Intratumor T helper type 2 cell infiltrate correlates with cancerassociated fibroblast thymic stromal lymphopoietin production and reduced survival in pancreatic cancer. The Journal of experimental medicine 208, 469-478

26 Fukuda, A., et al. (2011) Stat3 and MMP7 contribute to pancreatic ductal adenocarcinoma initiation and progression. Cancer Cell 19, 441-455

27 Sanford, D.E., et al. (2013) Inflammatory monocyte mobilization decreases patient survival in pancreatic cancer: a role for targeting the CCL2/CCR2 axis. Clin. Cancer Res. 19, 3404-3415 28 Tan, M.C., et al. (2009) Disruption of CCR5-dependent homing of regulatory T cells inhibits tumor growth in a murine model of pancreatic cancer. J. Immunol. 182, 1746-1755

29 Chao, T., et al. (2016) CXCR2-Dependent Accumulation of Tumor-Associated Neutrophils Regulates T-cell Immunity in Pancreatic Ductal Adenocarcinoma. Cancer Immunol Res 4, 968982

30 Demir, I.E., et al. (2017) Early pancreatic cancer lesions suppress pain through CXCL12mediated chemoattraction of Schwann cells. Proc. Natl. Acad. Sci. U. S. A. 114, E85-E94 31 Bayne, L.J., et al. (2012) Tumor-derived granulocyte-macrophage colony-stimulating factor regulates myeloid inflammation and T cell immunity in pancreatic cancer. Cancer Cell 21, 822835

32 Pylayeva-Gupta, Y., et al. (2012) Oncogenic Kras-induced GM-CSF production promotes the development of pancreatic neoplasia. Cancer Cell 21, 836-847

33 Lesina, M., et al. (2011) Stat3/Socs3 activation by IL-6 transsignaling promotes progression of pancreatic intraepithelial neoplasia and development of pancreatic cancer. Cancer Cell 19, 456-469

34 Daniluk, J., et al. (2012) An NF-kappaB pathway-mediated positive feedback loop amplifies Ras activity to pathological levels in mice. J. Clin. Invest. 122, 1519-1528

35 Sun, X., et al. (2010) CXCL12 / CXCR4 / CXCR7 chemokine axis and cancer progression. Cancer Metastasis Rev. 29, 709-722

36 Shakir, M., et al. (2015) The chemokine receptors CXCR4/CXCR7 and their primary heterodimeric ligands CXCL12 and CXCL12/high mobility group box 1 in pancreatic cancer growth and development: finding flow. Pancreas 44, 528-534

37 Singh, A.P., et al. (2012) CXCL12/CXCR4 protein signaling axis induces sonic hedgehog expression in pancreatic cancer cells via extracellular regulated kinase- and Akt kinase-mediated activation of nuclear factor kappaB: implications for bidirectional tumor-stromal interactions. $J$. Biol. Chem. 287, 39115-39124

38 Feig, C., et al. (2013) Targeting CXCL12 from FAP-expressing carcinoma-associated fibroblasts synergizes with anti-PD-L1 immunotherapy in pancreatic cancer. Proc. Natl. Acad. Sci. U. S. A. 110, 20212-20217

39 Zlotnik, A. and Yoshie, O. (2012) The chemokine superfamily revisited. Immunity 36, 705716

40 Varol, C., et al. (2015) Macrophages: development and tissue specialization. Annu. Rev. Immunol. 33, 643-675

41 Chang, J.H., et al. (2016) Role of immune cells in pancreatic cancer from bench to clinical application: An updated review. Medicine (Baltimore) 95, e5541

42 Liou, G.Y., et al. (2013) Macrophage-secreted cytokines drive pancreatic acinar-to-ductal metaplasia through NF-kappaB and MMPs. J. Cell Biol. 202, 563-577 
43 Ruffell, B., et al. (2012) Differential macrophage programming in the tumor microenvironment. Trends Immunol. 33, 119-126

44 Ireland, L., et al. (2016) Chemoresistance in Pancreatic Cancer Is Driven by Stroma-Derived Insulin-Like Growth Factors. Cancer Res. 76, 6851-6863

45 Weizman, N., et al. (2014) Macrophages mediate gemcitabine resistance of pancreatic adenocarcinoma by upregulating cytidine deaminase. Oncogene 33, 3812-3819

46 Seifert, L., et al. (2016) Radiation Therapy Induces Macrophages to Suppress T-Cell

Responses Against Pancreatic Tumors in Mice. Gastroenterology 150, 1659-1672 e1655 47 Nielsen, S.R., et al. (2016) Macrophage-secreted granulin supports pancreatic cancer metastasis by inducing liver fibrosis. Nat. Cell Biol. 18, 549-560

48 Beatty, G.L., et al. (2011) CD40 agonists alter tumor stroma and show efficacy against pancreatic carcinoma in mice and humans. Science 331, 1612-1616

$49 \mathrm{Zhu}, \mathrm{Y}$., et al. (2014) CSF1/CSF1R blockade reprograms tumor-infiltrating macrophages and improves response to T-cell checkpoint immunotherapy in pancreatic cancer models. Cancer Res. 74, 5057-5069

50 Clark, C.E., et al. (2007) Dynamics of the immune reaction to pancreatic cancer from inception to invasion. Cancer Res. 67, 9518-9527

51 Ene-Obong, A., et al. (2013) Activated pancreatic stellate cells sequester CD8+ T cells to reduce their infiltration of the juxtatumoral compartment of pancreatic ductal adenocarcinoma. Gastroenterology 145, 1121-1132

52 Daley, D., et al. (2016) gammadelta T Cells Support Pancreatic Oncogenesis by Restraining alphabeta T Cell Activation. Cell 166, 1485-1499 e1415

53 Zhang, Y., et al. (2017) Myeloid cells are required for PD-1/PD-L1 checkpoint activation and the establishment of an immunosuppressive environment in pancreatic cancer. Gut 66, 124-136 54 Lim, S.O., et al. (2016) Deubiquitination and Stabilization of PD-L1 by CSN5. Cancer Cell 30, 925-939

55 Pergamo, M. and Miller, G. (2016) Myeloid-derived suppressor cells and their role in pancreatic cancer. Cancer Gene Ther.

56 Ugel, S., et al. (2015) Tumor-induced myeloid deviation: when myeloid-derived suppressor cells meet tumor-associated macrophages. J. Clin. Invest. 125, 3365-3376

57 Tassi, E., et al. (2008) Carcinoembryonic antigen-specific but not antiviral CD4+ T cell immunity is impaired in pancreatic carcinoma patients. J. Immunol. 181, 6595-6603

58 Demols, A., et al. (2000) CD4(+ )T cells play an important role in acute experimental pancreatitis in mice. Gastroenterology 118, 582-590

59 Zhang, Y., et al. (2014) CD4+ T lymphocyte ablation prevents pancreatic carcinogenesis in mice. Cancer Immunol Res 2, 423-435

60 McAllister, F., et al. (2014) Oncogenic Kras activates a hematopoietic-to-epithelial IL-17 signaling axis in preinvasive pancreatic neoplasia. Cancer Cell 25, 621-637

61 Tang, Y., et al. (2014) An increased abundance of tumor-infiltrating regulatory T cells is correlated with the progression and prognosis of pancreatic ductal adenocarcinoma. PLoS One 9, e91551

62 Folias, A.E., et al. (2014) Aberrant innate immune activation following tissue injury impairs pancreatic regeneration. PLoS One 9, e102125

63 Rao, R., et al. (2014) Interleukin 17-producing gammadeltaT cells promote hepatic regeneration in mice. Gastroenterology 147, 473-484 e472 
64 Xue, J., et al. (2012) Aryl hydrocarbon receptor regulates pancreatic IL-22 production and protects mice from acute pancreatitis. Gastroenterology 143, 1670-1680

65 Xue, J., et al. (2016) Aryl Hydrocarbon Receptor Ligands in Cigarette Smoke Induce

Production of Interleukin-22 to Promote Pancreatic Fibrosis in Models of Chronic Pancreatitis.

Gastroenterology 151, 1206-1217

66 Pylayeva-Gupta, Y., et al. (2016) IL35-Producing B Cells Promote the Development of Pancreatic Neoplasia. Cancer Discov. 6, 247-255

67 Gunderson, A.J., et al. (2016) Bruton Tyrosine Kinase-Dependent Immune Cell Cross-talk

Drives Pancreas Cancer. Cancer Discov. 6, 270-285

68 Oberg, H.H., et al. (2014) Novel bispecific antibodies increase gammadelta T-cell

cytotoxicity against pancreatic cancer cells. Cancer Res. 74, 1349-1360

69 Neesse, A., et al. (2015) Stromal biology and therapy in pancreatic cancer: a changing paradigm. Gut 64, 1476-1484

70 Whatcott, C.J., et al. (2015) Desmoplasia in Primary Tumors and Metastatic Lesions of Pancreatic Cancer. Clin. Cancer Res. 21, 3561-3568

71 Erez, N., et al. (2010) Cancer-Associated Fibroblasts Are Activated in Incipient Neoplasia to Orchestrate Tumor-Promoting Inflammation in an NF-kappaB-Dependent Manner. Cancer Cell $17,135-147$

72 Neesse, A., et al. (2011) Stromal biology and therapy in pancreatic cancer. Gut 60, 861-868

73 Rhim, A.D., et al. (2014) Stromal elements act to restrain, rather than support, pancreatic ductal adenocarcinoma. Cancer Cell 25, 735-747

74 Sherman, M.H., et al. (2014) Vitamin D receptor-mediated stromal reprogramming suppresses pancreatitis and enhances pancreatic cancer therapy. Cell 159, 80-93

75 DuFort, C.C., et al. (2016) Mounting Pressure in the Microenvironment: Fluids, Solids, and Cells in Pancreatic Ductal Adenocarcinoma. Gastroenterology 150, 1545-1557 e1542

76 Aiello, N.M., et al. (2016) Metastatic progression is associated with dynamic changes in the local microenvironment. Nat Commun 7, 12819

77 Costa-Silva, B., et al. (2015) Pancreatic cancer exosomes initiate pre-metastatic niche formation in the liver. Nat. Cell Biol. 17, 816-826

78 Kruger, A. (2015) Premetastatic niche formation in the liver: emerging mechanisms and mouse models. J. Mol. Med. (Berl.) 93, 1193-1201

79 Diakopoulos, K.N., et al. (2015) Impaired autophagy induces chronic atrophic pancreatitis in mice via sex- and nutrition-dependent processes. Gastroenterology 148, 626-638 e617

80 Antonucci, L., et al. (2015) Basal autophagy maintains pancreatic acinar cell homeostasis and protein synthesis and prevents ER stress. Proc. Natl. Acad. Sci. U. S. A. 112, E6166-6174

81 Dumartin, L., et al. (2016) ER stress protein AGR2 precedes and is involved in the regulation of pancreatic cancer initiation. Oncogene

82 Guo, J.Y., et al. (2011) Activated Ras requires autophagy to maintain oxidative metabolism and tumorigenesis. Genes Dev. 25, 460-470

83 Ying, H., et al. (2012) Oncogenic Kras maintains pancreatic tumors through regulation of anabolic glucose metabolism. Cell 149, 656-670

84 Tape, C.J., et al. (2016) Oncogenic KRAS Regulates Tumor Cell Signaling via Stromal Reciprocation. Cell 165, 910-920

85 Sousa, C.M., et al. (2016) Pancreatic stellate cells support tumour metabolism through autophagic alanine secretion. Nature 536, 479-483 
86 Philip, B., et al. (2013) A high-fat diet activates oncogenic Kras and COX2 to induce development of pancreatic ductal adenocarcinoma in mice. Gastroenterology 145, 1449-1458 87 Incio, J., et al. (2016) Obesity-Induced Inflammation and Desmoplasia Promote Pancreatic Cancer Progression and Resistance to Chemotherapy. Cancer Discov. 6, 852-869 88 Meyer, K.A., et al. (2016) Adipocytes promote pancreatic cancer cell proliferation via glutamine transfer. Biochem Biophys Rep 7, 144-149

89 Flint, T.R., et al. (2016) Tumor-Induced IL-6 Reprograms Host Metabolism to Suppress Antitumor Immunity. Cell Metab 24, 672-684

90 Greco, S.H., et al. (2015) TGF-beta Blockade Reduces Mortality and Metabolic Changes in a Validated Murine Model of Pancreatic Cancer Cachexia. PLoS One 10, e0132786

91 Khorana, A.A. and Fine, R.L. (2004) Pancreatic cancer and thromboembolic disease. Lancet Oncol. 5, 655-663

92 Varki, A. (2007) Trousseau's syndrome: multiple definitions and multiple mechanisms. Blood $110,1723-1729$

93 Qi, Q., et al. (2015) Hyperfibrinogen Is Associated With the Systemic Inflammatory

Response and Predicts Poor Prognosis in Advanced Pancreatic Cancer. Pancreas 44, 977-982 94 Davalos, D. and Akassoglou, K. (2012) Fibrinogen as a key regulator of inflammation in disease. Semin. Immunopathol. 34, 43-62

95 Kitamura, T., et al. (2015) Immune cell promotion of metastasis. Nat. Rev. Immunol. 15, 7386

96 Massague, J. and Obenauf, A.C. (2016) Metastatic colonization by circulating tumour cells. Nature 529, 298-306

97 Connolly, M.K., et al. (2010) Distinct populations of metastases-enabling myeloid cells expand in the liver of mice harboring invasive and preinvasive intra-abdominal tumor. J. Leukoc. Biol. 87, 713-725

98 Seubert, B., et al. (2015) Tissue inhibitor of metalloproteinases (TIMP)-1 creates a premetastatic niche in the liver through SDF-1/CXCR4-dependent neutrophil recruitment in mice. Hepatology 61, 238-248

99 Grunwald, B., et al. (2016) Pancreatic Premalignant Lesions Secrete Tissue Inhibitor of Metalloproteinases-1, Which Activates Hepatic Stellate Cells Via CD63 Signaling to Create a Premetastatic Niche in the Liver. Gastroenterology 151, 1011-1024 e1017

100 McAllister, S.S. and Weinberg, R.A. (2014) The tumour-induced systemic environment as a critical regulator of cancer progression and metastasis. Nat. Cell Biol. 16, 717-727

101 Rodier, F. and Campisi, J. (2011) Four faces of cellular senescence. J. Cell Biol. 192, 547556

102 Linkermann, A., et al. (2014) Regulated cell death and inflammation: an auto-amplification loop causes organ failure. Nat. Rev. Immunol. 14, 759-767

103 Vesely, M.D., et al. (2011) Natural innate and adaptive immunity to cancer. Annu. Rev. Immunol. 29, 235-271

104 Mace, T.A., et al. (2013) Pancreatic cancer-associated stellate cells promote differentiation of myeloid-derived suppressor cells in a STAT3-dependent manner. Cancer Res. 73, 3007-3018 105 Hwang, R.F., et al. (2008) Cancer-associated stromal fibroblasts promote pancreatic tumor progression. Cancer Res. 68, 918-926

106 Richards, K.E., et al. (2016) Cancer-associated fibroblast exosomes regulate survival and proliferation of pancreatic cancer cells. Oncogene 
107 Martinez-Bosch, N., et al. (2014) Galectin-1 drives pancreatic carcinogenesis through stroma remodeling and Hedgehog signaling activation. Cancer Res. 74, 3512-3524

108 Tang, D., et al. (2012) High expression of Galectin-1 in pancreatic stellate cells plays a role in the development and maintenance of an immunosuppressive microenvironment in pancreatic cancer. Int. J. Cancer 130, 2337-2348

109 Leca, J., et al. (2016) Cancer-associated fibroblast-derived annexin A6+ extracellular vesicles support pancreatic cancer aggressiveness. J. Clin. Invest. 126, 4140-4156

110 Froeling, F.E., et al. (2011) Retinoic acid-induced pancreatic stellate cell quiescence reduces paracrine Wnt-beta-catenin signaling to slow tumor progression. Gastroenterology 141, 14861497, 1497 e1481-1414 


\section{Key Figure}

Figure 1

A

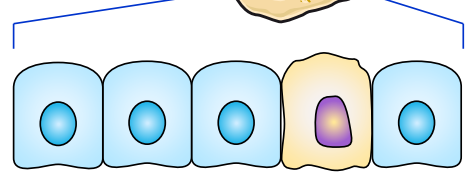

B

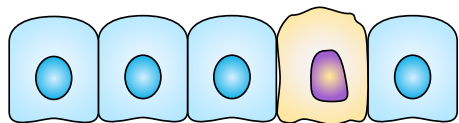

c

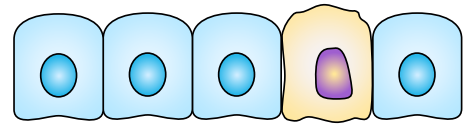

D

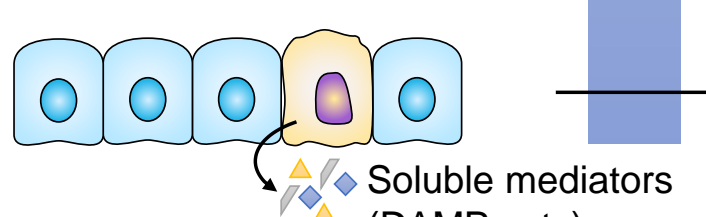

E

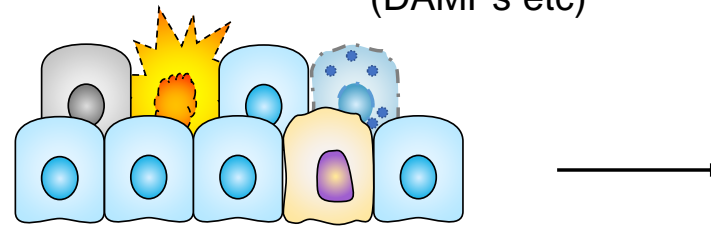

INSULT

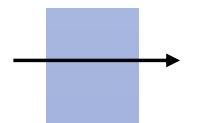

000000

CELL

Repair

\section{OUTCOME}

INFLAMMATION

TISSUE/ORGAN
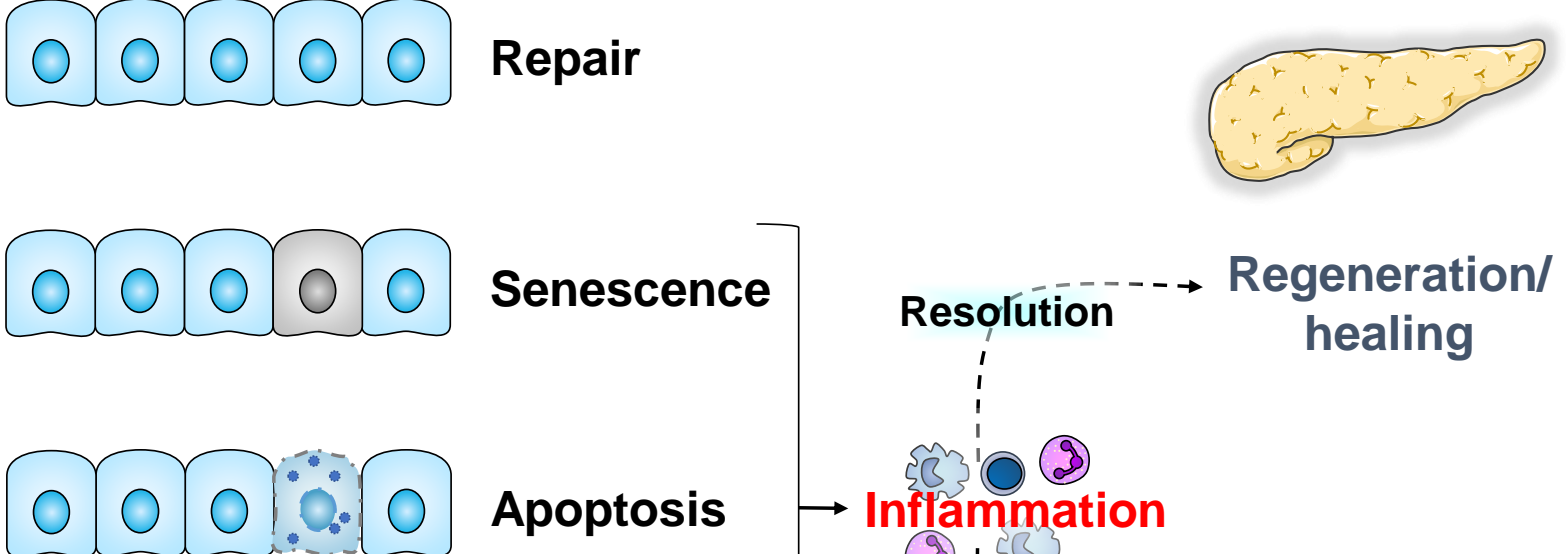

Apoptosis

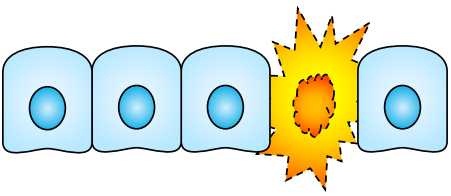

Necrosis/

Necroptosis

Resolution

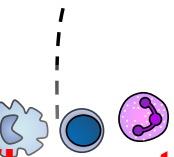

$\rightarrow$ Inflammation

(3) ! 追<smiles>[AlH][TlH]</smiles>

Persistence

Fibrosis

$\pm$

loss of function

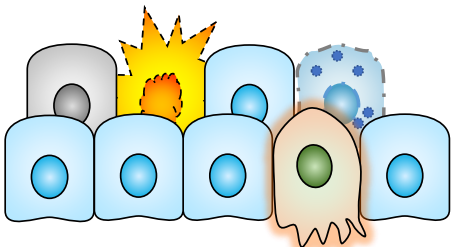

Malignant

transformation

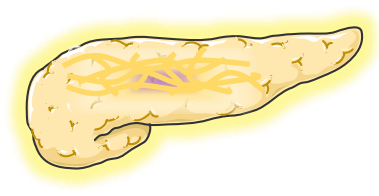

Immune system activation

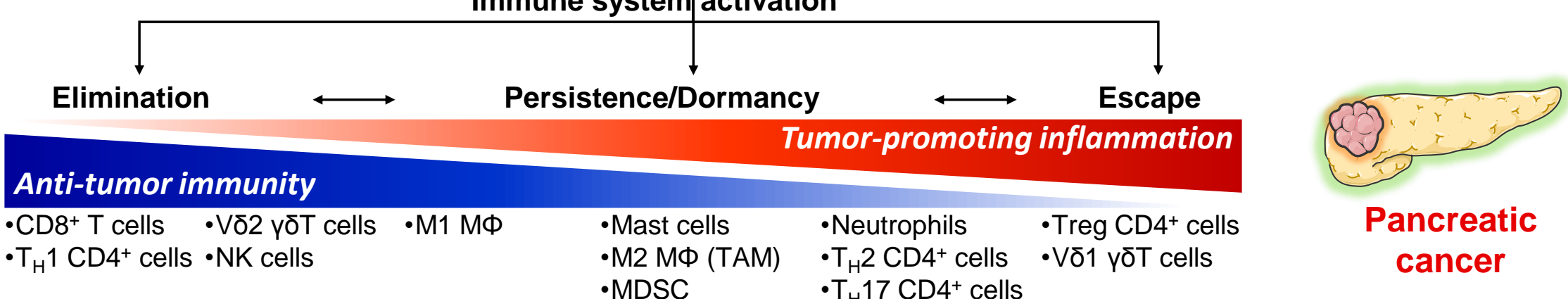




\section{Figure 2}

A

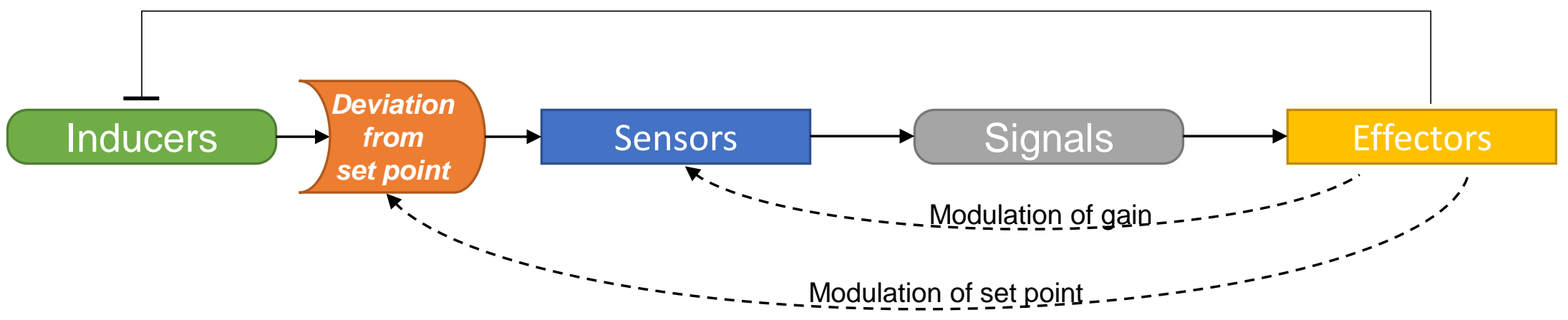

B

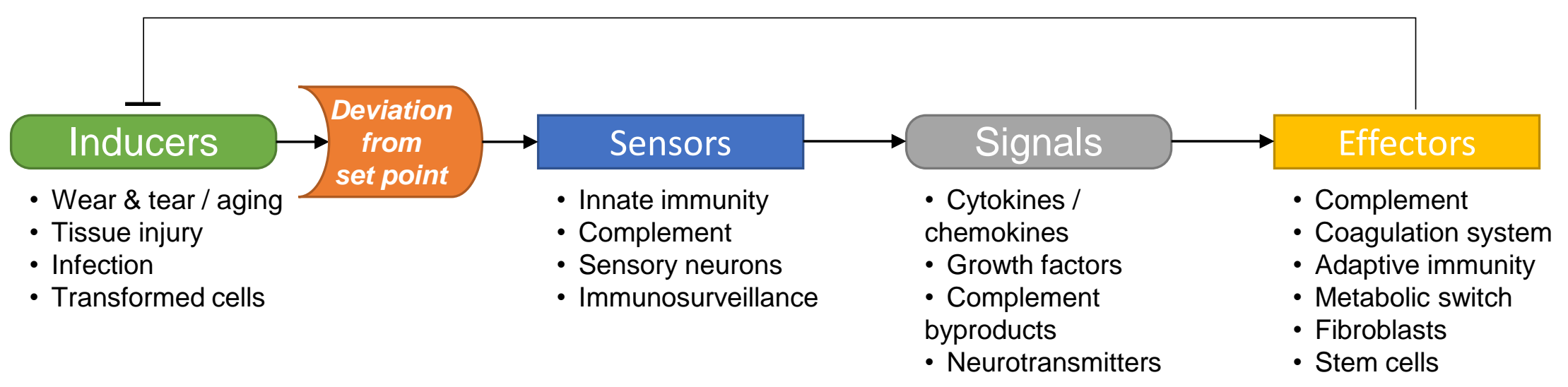


Figure 3
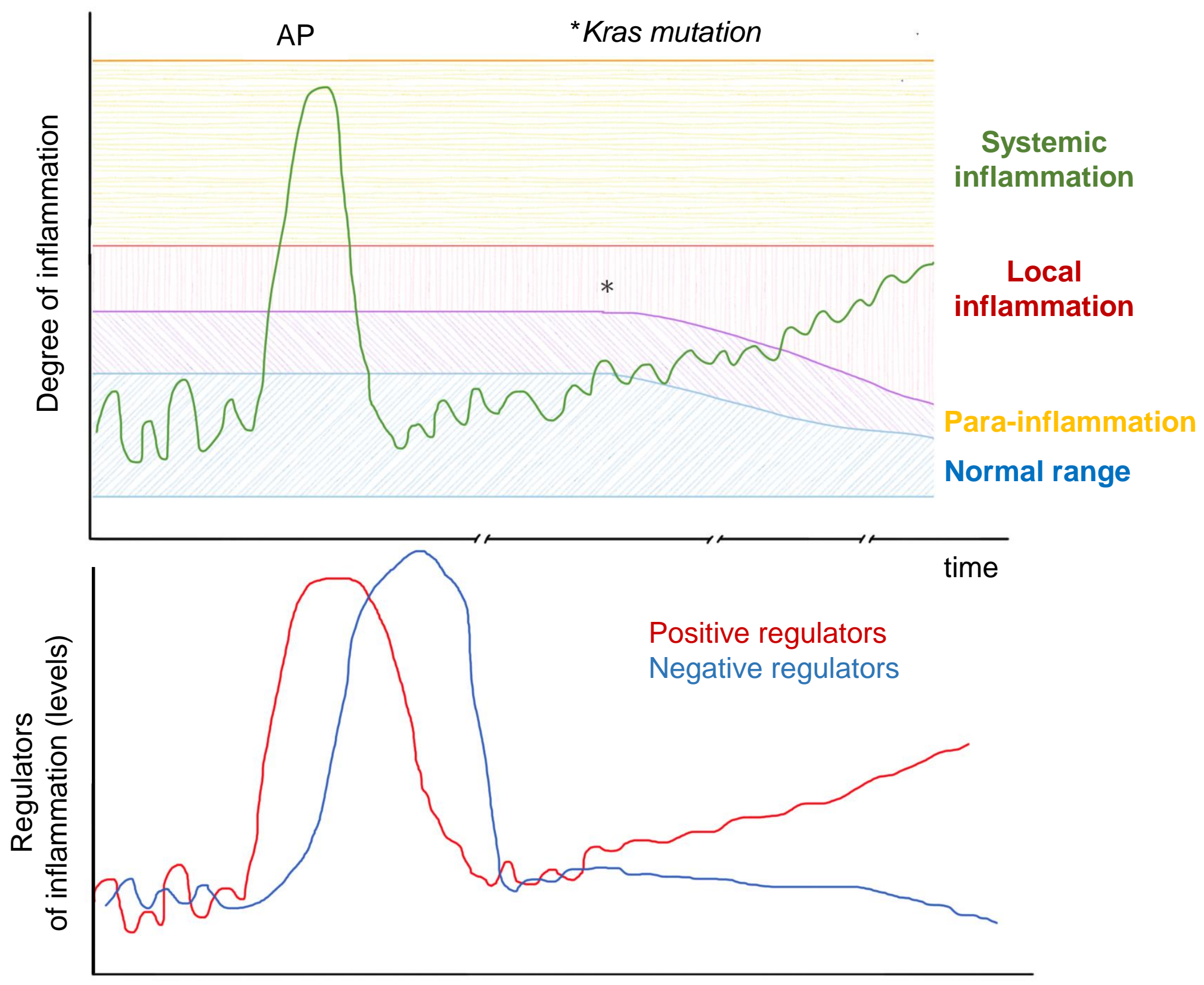

time 
Figure 4

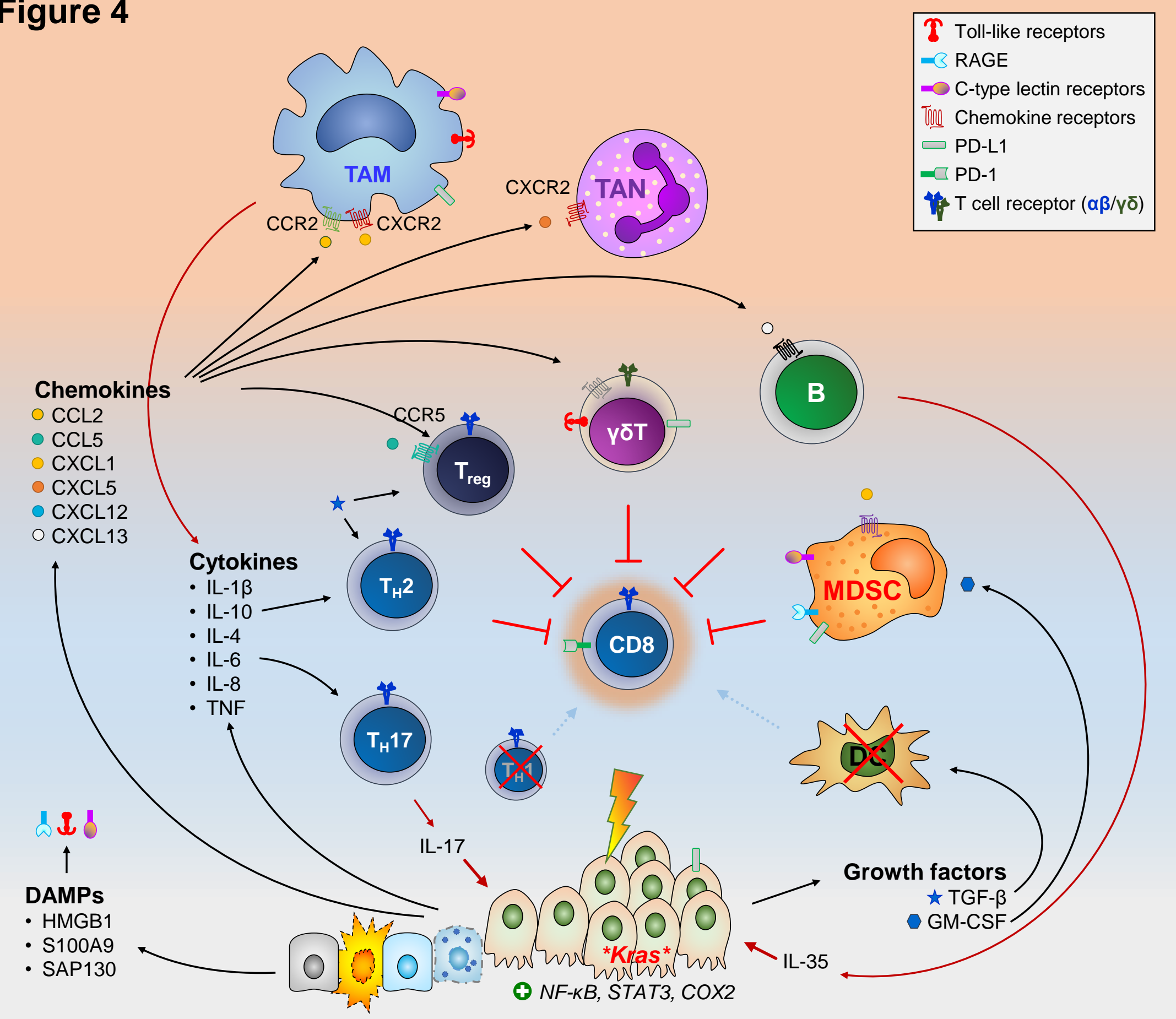




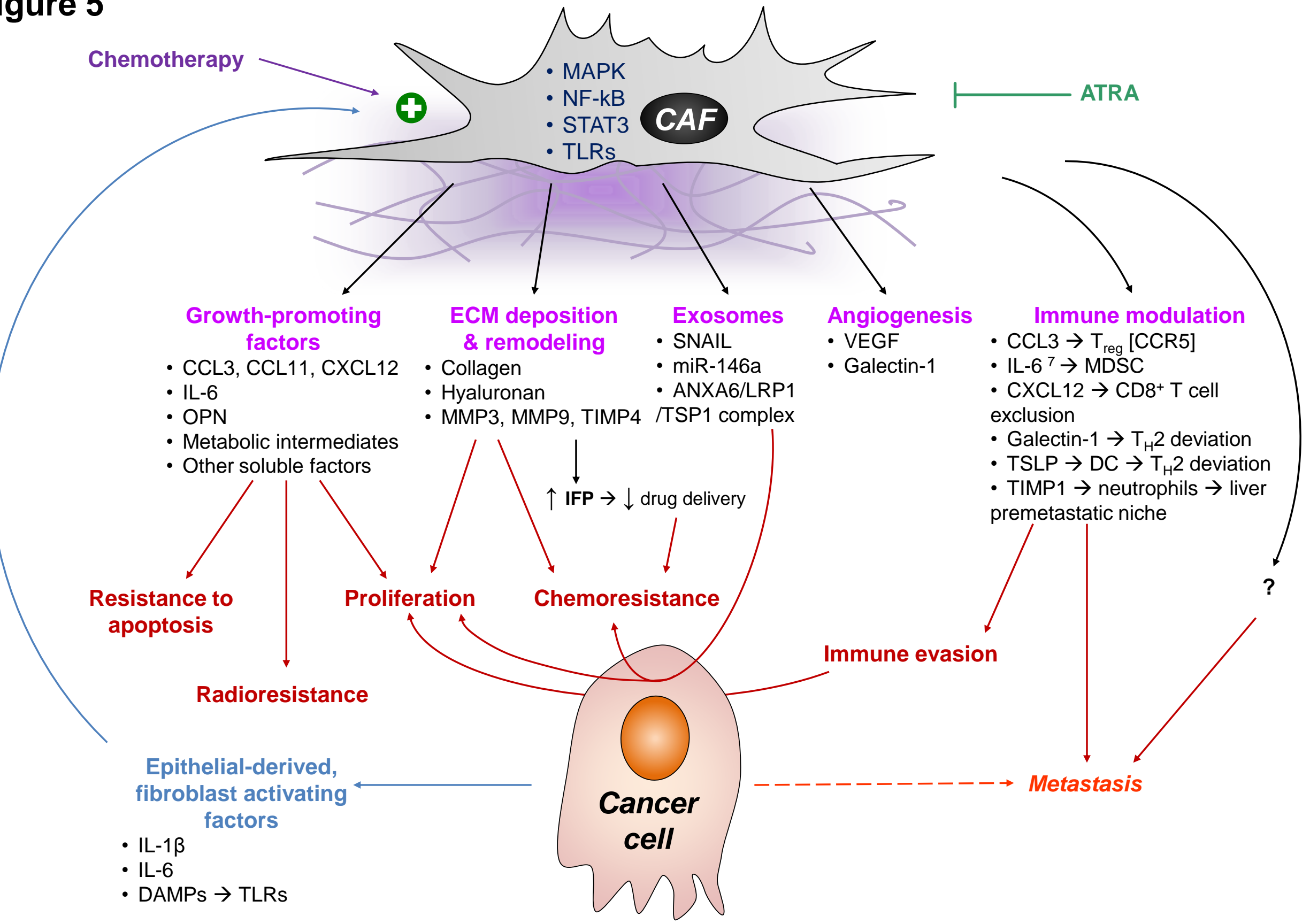

